\title{
Effectiveness of brace treatment: a systematic review
}

\author{
Toru Maruyama*, Yusuke Nakao, Katsushi Takeshita \\ From 7th International Conference on Conservative Management of Spinal Deformities \\ Montreal, Canada. 20-22 May 2010
}

\section{Introduction}

Despite the wide use of bracing for the treatment of adolescent idiopathic scoliosis (AIS), its effectiveness remains controversial. The purpose of this study was to investigate effectiveness of the bracing in the treatment of AIS through systematic review of the literature.

\section{Materials and methods}

A computer aided search of Pub Med database was performed using the keywords, <scoliosis $>$ and $<$ brace $>$ in September 2009. From the retrieved abstracts, studies comparing the results of brace treatment for AIS with no-treatment, other conservative treatments, or surgery were selected. Extracted outcomes including radiological curve progression, incidence of surgery, and quality of life (QOL) were analyzed.

\section{Results}

From a total of 1133 abstracts, 20 studies were finally selected. There were no randomized controlled trial, 2 nonrandomized clinical controlled trials (CCT) and 18 retrospective case-control studies.

One CCT demonstrated lower failure rate with bracing than with observation in girls with Cobb angle of 25 to $35^{\circ}$. Another CCT with a follow-up period of 16 years showed lower progression rate and less incidence of surgery with bracing than with observation in girls with Cobb angles around 30o. There was conflicting evidence that the bracing affects the QOL of the patients, compared with their observed counterparts.

One CCT demonstrated lower failure rate with bracing than with electrical stimulation in girls with Cobb angle of 25 to 35 degrees. An advantage of the bracing over side-shift exercise or casting was not certified.
QOL of the patients treated with brace was not different from those treated surgically, although comparison is difficult because in most of the studies curve magnitude at baseline was considerably larger in the surgery group.

\section{Discussion}

There was no high validation study comparing brace treatment with observation or other treatments. One main reason is the difficulty to conduct randomized controlled trials in the treatment of AIS. Wellconstructed prospective studies that employ historical controls for comparison may deserve more attention.

\section{Conclusion}

Although the evidence level is limited, bracing has more effect than observation or electrical stimulation to prevent progression and may not have a negative impact on patients of QOL. Therefore, bracing can be recommended for the treatment of AIS, at least for female patients with Cobb angle of 25 to 350 .

Published: 10 September 2010

doi:10.1186/1748-7161-5-S1-061

Cite this article as: Maruyama et al:: Effectiveness of brace treatment: a systematic review. Scoliosis 2010 5(Suppl 1):O61.

Saitama Medical Center, Saitama Medical University, Saitama, Japan

Full list of author information is available at the end of the article 